\title{
O Estado da Arte da Comunicação Organizacional
}

\author{
Rita Monteiro Mourão, Sandra Miranda \& Gisela Gonçalves \\ Universidade da Beira Interior / Escola Superior de Comunicação Social - Instituto \\ Politécnico de Lisboa / Universidade da Beira Interior \\ E-mail: ritaandreiamourao@gmail.com/smirandadescs.ipl.pt / \\ gisela.ubi@gmail.com
}

\begin{abstract}
RESUMO
A investigação em comunicação organizacional tem vindo a crescer nos últimos anos e a constituir-se como uma necessidade premente para a compreensão da dinâmica e do comportamento organizacional. Porém, o investimento nesta área de estudo e o entendimento da sua complexidade implica a identificação de um percurso histórico e das linhas de investigação que lhe são inerentes. O presente trabalho, tem como principal objetivo revisitar as diferentes linhas de investigação e os respetivos paradigmas que, historicamente, têm atravessado a investigação

em comunicação organizacional até aos nossos dias. Nesse sentido, serão identificados e explicitados os quatro paradigmas principais associados à comunicação organizacional, sendo estes: o paradigma positivista; o paradigma interpretativo; o paradigma crítico e o paradigma dialógico. Esta discussão - praticamente inexistente na literatura, torna-se fundamental não só para percebermos o contexto histórico da comunicação organizacional, mas também para elevarmos o estado de maturidade da disciplina providenciado uma perspetiva integrada da mesma.
\end{abstract}

Palavras-chave: comunicação organizacional; paradigmas; estado da arte.

Data de submissão: 26-04-2016. Data de aprovação: 23-10-2016.

Esta publicação foi apoiada pela Fundação para a Ciência e Tecnologia (FCT), pelo Fundo Social Europeu e fundos nacionais MEC no âmbito da bolsa individual de doutoramento com a referência PD/BD/114005/2015.

A Revista Estudos em Comunicação é financiada por Fundos FEDER através do Programa Operacional Factores de Competitividade - COMPETE e por Fundos Nacionais através da FCT - Fundação para a Ciência e a Tecnologia no âmbito do projeto Comunicação, Filosofia e Humanidades (LabCom.IFP) UID/CCI/00661/2013.

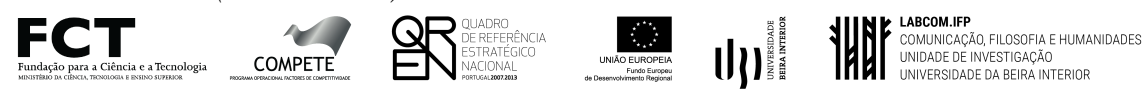




\begin{abstract}
The research about organizational communication has been growing in recent years, because it is necessary to understand the dynamics and organizational behavior. However, the investment in this area of study and understanding of its complexity takes into account the understanding of an historical background and research lines that are inherent. This article aims to revisit the different lines of research and the respective paradigms that historically have crossed the rese-

the present day. In this sense, the four main paradigms associated with organizational communication will be identified and explained, namely: the positivist paradigm; the interpretative paradigm; the critical paradigm and the dialogical paradigm. This discussion - which is little reported in the literature is essential, not only to realize the historical context of organizational communication, but also to understand the discipline mature state considering an integrated perspective.
\end{abstract} arch in organizational communication to

Keywords: organizational communication; paradigms; state of art.

\title{
INTRODUÇÃO
}

definição da comunicação organizacional, enquanto disciplina, tem vindo a constituir-se como uma necessidade (Ruão, 2004). Assim, o seu reconhecimento implica que tenhamos de recuar ao passado (Krone, 2007). Contudo, tal definição tem vindo a ser dificultada pelo facto da comunicação organizacional assumir, ainda, uma identidade pouco clara. As complexidades organizacionais, na qual a comunicação organizacional se enquadra e o seu recente estudo (i.e., remonta às últimas décadas do século $\mathrm{XX}$ ) contribuem para que a questão: O que é a comunicação organizacional? seja, muitas vezes, substituída por Como é que podemos encarar a comunicação organizacional, através de uma determinada perspetiva e em detrimento de uma outra? (Deetz, 2001; Mumby \& Stohl, 1996). Isto porque, sendo este tipo de comunicação um processo dinâmico e complexo, surge a necessidade de enquadrá-la no âmbito de diferentes linhas de investigação, tendo em conta, também, diferentes períodos históricos (Ruão 2004; Ruão, 2008).

De forma a sistematizar o quadro de investigação que envolve a comunicação organizacional, Deetz (2001), identifica as quatro principais tendências 
dos estudos de investigação sobre este tipo de comunicação, considerando o seu legado histórico interdisciplinar. Este autor defende a existência de um conjunto de alternativas, ou seja, um conjunto de sistemas linguísticos úteis para a investigação em comunicação organizacional. Assim, podemos considerar quatro paradigmas que orientam as organizações, a forma de gerir os seus públicos internos e de reportar os eventos organizacionais (SchockleyZalabak, 2012). Estes paradigmas poderão ser representados por um determinado quadrante (figura 1), sendo o eixo vertical constituído pelos extremos Dissensus e Consensus e o eixo horizontal representado pelos extremos Local/Emergent e Elite/A priori.

Segundo a perspetiva de Deetz (2001), cada uma das dimensões acima referidas, poderá ser definida da seguinte forma:

Local/Emergent - dá atenção aos investigadores que trabalham segundo uma linguagem aberta. Neste caso, são considerados sentimentos e intuições. O estudo é guiado mais pela formação de conceito do que pela sua aplicação. $\mathrm{O}$ investigador é encarado como um colaborador na produção de conhecimento ao invés de um expert observador;

Elite/A priori - tem em conta uma linguagem específica da comunidade científica. Neste caso, o processo de investigação considera definições a priori e já existe um conhecimento prévio, contrariamente ao que se verifica na dimensão Local/Emergent;

Consensus e Dissensus - estas expressões não indicam acordo/desacordo, remetendo antes para a unidade vs diferença, continuidade vs descontinuidade. A dimensão Consensus tende a ser encarada como algo normativo, natural e não interpretativo. Nesse âmbito, a linguagem tende a constituir-se como neutra e transparente, para uso partilhado, não podendo existir um desvio. Por sua vez, o conflito e a fragmentação são encarados como problemáticos. A dimensão relativa ao Dissensus remete-nos para aspetos relacionados com o conflito e a tensão. Neste caso, a investigação tende a ser encarada como algo conflitual, por si só, considerando-se uma variedade e alternativas.

Os quatro paradigmas são, cada um deles, constituídos por um dos extremos do eixo horizontal e um dos extremos do eixo vertical. Desta forma, Deetz (2001) considera o paradigma positivista, constituído pelo Consensus e pelo Elite/A priori; o paradigma interpretativo, constituído pelo Consensus e 
pelo Local/Emergent; o paradigma crítico, constituído pelo Dissensus e pelo Elite/a priori e, finalmente, o paradigma dialógico, constituído pelo Dissensus e o Local/Emergent. Para melhor compreensão, cada um dos paradigmas encontra-se identificado na imagem abaixo.

Figura 1. Representação dos 4 paradigmas da Comunicação Organizacional.

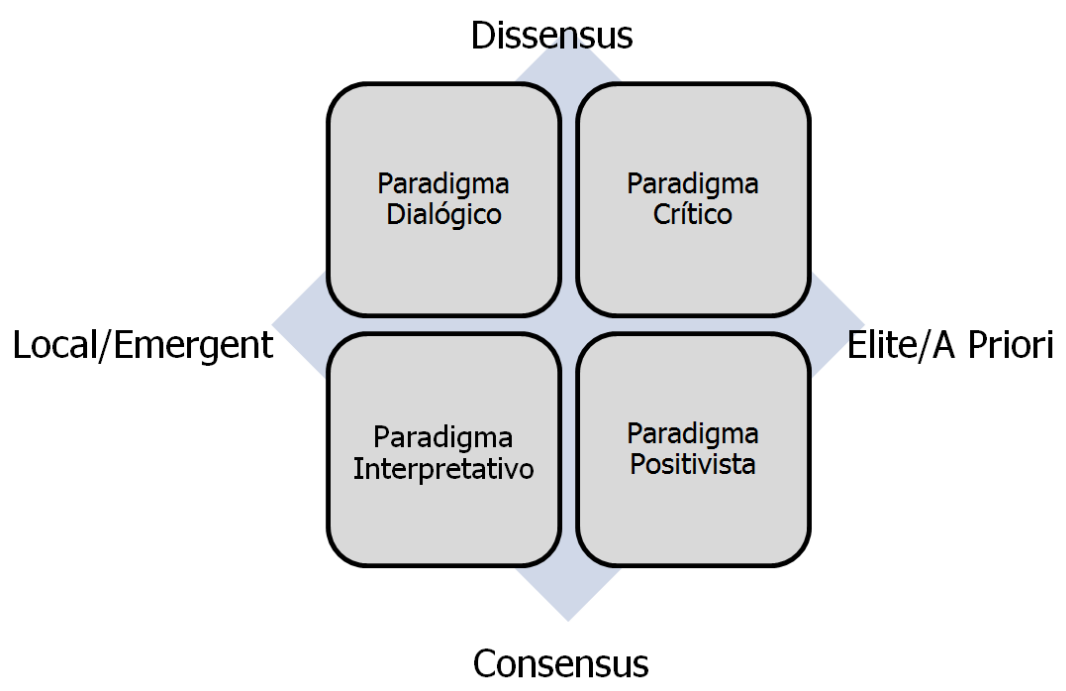

(adaptado de Deetz, 2001, p. 11)

Face ao referido anteriormente, os quatro paradigmas sobre a investigação no âmbito da comunicação organizacional serão explicitados em seguida. Neste caso, ter-se-á em conta não somente os argumentos defendidos por Deetz (2001), como também por outros autores responsáveis pelo estudo da comunicação organizacional enquanto disciplina.

\section{O Estado da Arte da Comunicação Organizacional}

\section{Paradigma Positivista: O discurso dos estudos normativos}

O estudo da comunicação organizacional teve o seu maior enfoque a partir do ano de 1940, dando os seus primeiros passos enquanto disciplina científica entre os anos de 1942 e 1947, designados por Seminal Years (Redding, 1985). 
Contudo, até aos anos 60 não existia um campo próprio de comunicação organizacional, sendo esta muitas vezes associada a disciplinas como a psicologia, a sociologia e a gestão (Redding, 1985; Ruão, 2004). Assim, os seus primeiros estudos realizaram-se entre os anos de 1900 e 1970, designando-se este período por período positivista ou de emancipação (Ruão, 1999).

O paradigma positivista encara as organizações como algo natural e aberto à descrição, à predição e ao controlo, sendo os seus estudos marcados pelo "princípio da racionalidade" (Mumby \& Stohl, 1996). Segundo este princípio, os estudos de comunicação organizacional baseiam-se, essencialmente, num conhecimento instrumental que privilegie a predição e o controlo, com vista à eficácia organizacional (Deetz, 2001; Mumby \& Stohl, 1996; Ruão, 1999; Spence \& Baker, 2007; Taylor, 1993).

A designação de discurso dos estudos normativos, associada ao paradigma positivista, advém do facto de considerar a normalização dos fenómenos comunicativos. Neste caso, assume-se que os objetivos de um grupo específico tendem a ser encarados como os objetivos da própria organização. Nesse âmbito, a organização é encarada como algo pré-existente com determinados fins instrumentais (i.e., gerar dinheiro/lucro), desconsiderando-se, portanto, outros fins organizacionais (e.g. motivação) e as posições dos membros (Deetz, 2001; Redding \& Tompkins, 1988; Schockley-Zalabak, 2012; Taylor, 1993).

A investigação realizada a partir do paradigma positivista, tem em conta as práticas convencionais e os determinantes metodológicos, considerando, por isso, a cobertura de determinadas leis. Neste caso, os objetos construídos através das práticas têm de ser constantes e permanentes. Portanto, têm-se em linha de conta conceções existentes a priori e factos como cómodos. Este paradigma é, também, tendencialmente conhecido como funcionalista, porque utiliza a análise tradicional de variáveis, com vista a generalizações dos fenómenos observados (Deetz, 2001; Redding \& Tompkins, 1988; Ruão, 1999; Wrench \& Punyanunt-Carter, 2012).

No que concerne à comunicação, esta é representada em termos de informação e de administração. Assim, a investigação centra-se, essencialmente, na resolução de problemas relativos à ineficiência comunicativa, sendo as metodologias de investigação utilizadas de cariz, essencialmente, quantitativo (Deetz, 2001; Miller, 2001; Spence \& Baker, 2007). Especificamente, importa frisar que a comunicação organizacional tende a ser encarada como uma transmissão e uma retroação. Assim, o paradigma positivista pressupõe um modelo 
telegráfico deste tipo de comunicação, pois os indivíduos respondem de forma mecânica aos estímulos externos, sendo essencialmente reativos. Por sua vez, a unidade de análise trata-se da entidade organizacional, considerando-se as suas caraterísticas sociais, psicológicas e económicas que são percecionadas como estáticas e não como processos sociais. Nesse sentido, a organização é encarada como uma estrutura concreta e a comunicação como uma substância tangível (Bouzon, 2013). Este tipo de estudos descura, então, as relações estabelecidas no âmbito organizacional. Isto porque estas relações implicam questões subjetivas que não podem ser interpretadas através dos discursos normativos, uma vez que, para isso, seriam necessárias não somente observações, como também entrevistas e questionários auto reportados (Deetz, 2001).

Torna-se relevante referir que o paradigma positivista encontra-se marcado, também, pelo reconhecimento académico da comunicação organizacional. É durante este período que este tipo de comunicação começa a solidificarse enquanto disciplina, diferenciando-se da psicologia, da sociologia e da gestão (Spence \& Baker, 2007). Para além disso, é durante esta época que surgem as primeiras publicações científicas e o primeiro curso de licenciatura (em 1959 - ano de cristalização), bem como a primeira conferência especializada (realizada em 1967 - ano de aceitação oficial) (Redding \& Tompkins, 1988; Ruão, 2008; Taylor \& Trujillo, 2001).

Entre os anos de 1960/1970 o paradigma positivista entra em declínio. Tal deve-se, a algumas limitações deste tipo de abordagem. A primeira limitação refere-se ao facto, das observações serem, inevitavelmente, influenciadas pela posição teórica do investigador, contrariamente à verdade absoluta defendida pelos positivistas. Para além disso, o facto do paradigma positivista ser determinista (i.e., a crença de que os resultados e os fenómenos são o resultado de causas que podem ser duplicadas); reducionista (i.e., o objetivo reduz-se ao fenómeno em si para ser mais fácil estudá-lo); preditivo (i.e., o seu principal objetivo é predizer comportamentos) e empírico (i.e., apenas os dados que se observam podem ser analisados) também se torna alvo de discussão. Assim, a necessidade de distanciar a comunicação organizacional dos estudos de gestão (todos os fenómenos podem ser numericamente medidos) e a necessidade de considerar o holismo, ao invés do atomismo e a causalidade dinâmica, em detrimento da causalidade unidirecional, remete-nos para o surgimento de perspetivas alternativas, surgindo o paradigma interpretativo (Deetz, 2001; Jones, Watson, Gardner \& Gallois, 2004; Krone, 2007; Mumby \& Stohl, 1996). 


\section{Paradigma Interpretativo: $O$ discurso dos estudos interpretativos}

A relevância dada ao paradigma interpretativo torna-se exponencial a partir dos anos 80, ficando conhecido este período, também, como momento de maturidade e de inovação (Redding \& Tompkins, 1988).

De acordo com o paradigma interpretativo, as organizações são encaradas como simbólicas, estruturais e pluralistas. Ou seja, são simbólicas, porque representam relações no processo de mudança; são estruturais, uma vez que podem ter efeitos nas ações diárias dos seus membros; são encaradas como pluralistas, contrariamente à visão unitária da escola funcionalista, porque podem ser compostas por grupos com diferentes interesses e objetivos (Bouzon, 2013). Nesse sentido, para os investigadores interpretativos, a organização trata-se de um espaço social, assumindo-se por isso, como um tipo específico de comunidade que pode partilhar caraterísticas com outras comunidades. Neste caso, o paradigma interpretativo dá ênfase ao cariz social, em detrimento da visão económica das atividades organizacionais, defendida no paradigma positivista. O principal objetivo é o de perceber como é que as realidades particulares, no seio organizacional são produzidas e mantidas ao nível da oralidade, das histórias, dos ritos e rituais e de outras atividades diárias. Ou seja, os discursos interpretativos são, tendencialmente, mais abertos e emergentes e menos ligados à eficiência e à produtividade quando comparados com o paradigma positivista. Nesse âmbito, os indivíduos deixam de ser defrontados como objetos (tratados como todos os outros) e passam a ser encarados como seres ativos, tal como o investigador (Deetz, 2001).

No caso específico da comunicação, pretende perceber-se como é que as pessoas comunicam no seu ambiente natural, contrariamente ao contexto "laboratorial" defendido pelos positivistas (Yanow \& Ybema, 2009). Assim, esta deixa de estar associada à simples transmissão de informação. Isto porque se verifica um interesse em ambas as partes. Ou seja, tem-se em consideração quem dá e quem recebe a informação (i.e., meta-comunicação). Especificamente, pretende compreender-se como é que os indivíduos percebem os seus próprios comportamentos comunicativos e qual o significado que dão a esses mesmos comportamentos (Wrench \& Punyanunt-Carter, 2012).

Por esse motivo, o paradigma interpretativo ficou, também, conhecido por meaning-centered approach (Schockley-Zalabak, 2012), sublinhando-se as dimensões simbólicas e expressivas das organizações, como é o caso da 
cultura organizacional. Nesse âmbito, as organizações passam a ser encaradas como unidades de partilha de valores, práticas e significados, assumindo-se a comunicação não somente como mais uma atividade organizacional, mas antes como um processo subjetivo e socialmente construído e com consequências para as relações sociais (Blair, Roberts \& McKecnie, 1985; Bouzon, 2013; Putnam \& Pacanowsky, 1983; Schockley-Zalabak, 2012; Taylor, 1993). Este paradigma veio descrever a comunicação organizacional como o processo responsável por gerar realidades partilhadas que permitem a tomada de decisão, o consenso, a influência e a cultura, sendo todos estes conceitos encarados como processos de comunicação que ajudam a criar a interação humana (Schockley-Zalabak, 2012).

No que concerne aos métodos utilizados, verifica-se uma utilização dos métodos qualitativos através de estudos de caso, observação prolongada de contextos reais, utilizando-se a observação participante, entrevistas em profundidade e contacto pessoal. Isto porque os investigadores pretendem perceber como é que as culturas organizacionais se desenvolvem e modificam (Blair, Roberts \& McKecnie, 1985; Deetz, 2001; Taylor \& Trujillo, 2001).

O período relativo ao paradigma interpretativo fica marcado pela publicação do livro "The Social Psychology of Organizing" em que Karl Weick (1979) defende a ideia de que as organizações não podem existir sem que exista interação humana e, por isso, sem que exista comunicação. É a partir desta altura que os conceitos de comunicação e de organização passam a ser encarados como sinónimos (Schockley-Zalabak, 2012). Para além disso, esta época fica, também, marcada pelo crescimento da investigação e das premissas teóricas em comunicação organizacional, sendo a partir daí que a disciplina sofre um avultado enriquecimento concetual e metodológico. Tal crescimento deve-se, essencialmente, à concretização de uma conferência sobre as abordagens interpretativas alternativas aos modelos positivistas até então defendidos, realizada no ano de 1981 (Putnam \& Pacanowsky, 1983; Redding \& Tompkins, 1988; Ruão, 2008).

Apesar do enriquecimento concetual da comunicação organizacional, desenvolvido durante o período interpretativo, entre os anos de 1980 e 1990, esta disciplina inicia um processo de crise de legitimidade e de representação. Isto porque surge a necessidade de ter em conta outros aspetos, como por exemplo, como é que se gerem os conflitos no seio organizacional (Deetz, 2001). Neste 
momento, surgem as teorias críticas àquelas até então existentes e defendidas (Taylor \& Trujillo, 2001).

\section{Paradigma Crítico: Discurso dos estudos críticos}

Em 1980, a abordagem interpretativa começa a ser alvo de críticas, tendo em conta a intenção hegemónica de alguns processos comunicativos. Assim, em 1990 surge o paradigma crítico que provém da Escola de Frankfurt. No caso do paradigma crítico, os investigadores encaram as organizações como contextos histórico-sociais, espaços de cariz político e compostos por relações de poder, através dos quais será importante desenvolver teorias relacionadas com as tomadas de decisão (Deetz, 2001). O discurso do paradigma crítico tem em conta questões como: a dominação, a assimetria e a distorção da comunicação. Isto porque, segundo este paradigma, as pessoas, no seio organizacional, tendem a agir, consoante os seus próprios interesses (Alvesson \& Aschcraft, 2009; Deetz, 2001). Assim, estas formas de exercício de poder podem produzir efeitos negativos nas tomadas de decisão, gerando, posteriormente, conflitos organizacionais e, consequentemente, distorções comunicativas, rotinas, normalizações ou falsos consentimentos (Alvesson \& Aschcraft, 2009; Deetz, 2001; Ruão, 2008).

O paradigma crítico centra-se, então, em questões relacionadas com: as ideologias; as instituições; os interesses e as identidades. Nesse âmbito, o envolvimento destes quatro aspetos tem em conta uma ideia que passa a designar-se por Questionando os 4 I's. As ideologias referem-se a crenças, mitos e doutrinas que guiam os indivíduos, os grupos ou as organizações. Os teóricos das perspetivas críticas pretendem examinar até que ponto essas ideologias foram desenvolvidas na organização para serem prejudiciais para alguns (especialmente para os detentores de menor poder). As instituições dizem respeito às instituições dominantes na sociedade, de forma a perceber se estas agem de forma prejudicial para os trabalhadores. Os teóricos do paradigma crítico têm em conta os interesses, para perceber se são ou não incontestáveis e, ao mesmo tempo, prejudiciais para os membros com menos poder. Finalmente, têm-se em consideração as identidades, no sentido de compreender quais é que poderão ser, mais uma vez, prejudiciais para os trabalhadores (Alvesson \& Aschcraft, 2009). 
De acordo com Mumby e Stohl (1996): "a comunicação organizacional não é um processo neutro de transmissão de informação, sendo assim um processo de organização que pode acarretar consequências políticas" (p.60). Considerando os argumentos de Mumby e Stohl (1996), pode mencionar-se que em termos de comunicação organizacional, o principal objetivo do paradigma crítico tem a ver com a criação de sociedades e postos de trabalho livres de um determinado domínio e controlo, onde os membros possam contribuir, de igual forma, para o desenvolvimento da organização. Isto porque este paradigma tem em conta as questões de poder e de abuso de poder, em que existe um processo de controlo baseado num grupo dominante que leva os restantes a acreditar que a submissão é a norma (Mumby, 2001; Schockley-Zalabak, 2012).

No que respeita ao aspeto metodológico, importa referir que, neste caso, já existe uma teoria a priori. Porém, as metodologias utilizadas são as qualitativas e idênticas àquelas que são utilizadas no paradigma interpretativo (Alvesson \& Aschcraft, 2009; Deetz, 2001; Ruão, 2008) Ou seja, a observação de participantes, as entrevistas e os modelos diferenciados de abordagem de resultados (Deetz, 2001). Isto porque, tal como no caso do paradigma interpretativo, também o paradigma crítico tem em conta a subjetividade e a abertura à interpretação (Wrench, 2013).

Finalmente, torna-se pertinente frisar a existência de duas correntes associadas aos estudos críticos, sendo estas: a ideologia crítica e a ação comunicativa. A ideologia crítica surgiu com o autor Marx. Este autor verificou que existe uma coerção, relativamente a quem é o detentor do capital. Nesse âmbito, sugere-se que os interesses específicos falham na sua concretização, uma vez que as pessoas não têm capacidade suficiente para perceber e agir, de acordo com os seus próprios interesses. Portanto, o que se pretende será banir a coerção e promover a adoção de processos sistémicos. No caso da ação comunicativa, consideram-se os estudos sobre distorção comunicativa, do autor Habermas, realizados a partir dos anos 70. Segundo este autor, o ato comunicativo, para constituir-se eficaz terá de considerar não somente o indivíduo que "fala", como também o indivíduo que "escuta". Nesse âmbito são considerados quatro domínios da realidade: a linguagem, o mundo externo, as relações humanas e o mundo interno do indivíduo. O ato comunicativo terá, também, de ter em conta: a apresentação de uma expressão que seja compreendida; ter uma proposição de conhecimento; estabelecer relações sociais 
legítimas; ter em conta a posição/ experiência de quem fala. Estes aspetos podem ser a base de uma comunicação eficaz como, também, da distorção comunicativa. Ainda relativamente à ação comunicativa, importa referir que, para que a participação na resolução de conflitos se torne aberta e livre, têm de considerar-se determinadas condições, sendo estas as seguintes: a existência de uma distribuição simétrica de possibilidade de discurso; a interpretação livre do mundo, para que haja uma conceção e desenvolvimento social da verdade, sendo que o ideal será que os participantes tenham oportunidade de expressar as suas próprias interpretações, gerando-se conflitos resolúveis; os indivíduos deverão ter oportunidade de estabelecer relações sociais legítimas e normas de condução e interação. Assim, conclui-se que as responsabilidades e os direitos devem ser adquiridos, segundo uma negociação e interação. Face aos aspetos mencionados anteriormente, torna-se possível perceber que a ação comunicativa inclui uma conceção de comunicação participativa (i.e., através da qual é possível descrever a possibilidade de tomadas de decisão mútuas) (Deetz, 2001).

Finalmente, importa frisar o papel do paradigma dialógico. Este é idêntico ao discurso dos estudos críticos, embora tenha em conta os processos micropolíticos.

\section{Paradigma Dialógico: Abordagem pós-moderna}

O paradigma dialógico surge no âmbito do pós-modernismo, tendo sido a sua perspetiva realçada em França por autores como Bourdieu, Foucault, Mumby \& Putnam (1992) e Burrell (1988). Este paradigma é idêntico ao paradigma crítico, uma vez que tem em conta questões de assimetria e de poder no âmbito da comunicação organizacional. Contudo, realça o papel dos processos micro políticos, da fragmentação e da resistência (Deetz, 2001; Ruão, 2008). Neste caso, a coerção passa a ser encarada como algo de cariz fluido, situacional, sem um local próprio ou origem (Deetz, 2001). Considera-se que apesar de ter em conta uma perspetiva crítica, o paradigma dialógico tende a ser mais radical do que o paradigma crítico (Krone, 2007). Isto porque o paradigma crítico veio demonstrar situações de domínio e de produção de oposição. Por sua vez, o paradigma dialógico pretende desmascarar e desconstruir mitos e assunções, para abrir o leque de possibilidades de novos entendimentos e processos (Schockley-Zalabak, 2012). Assim, o paradigma dialógico 
baseia-se, sobretudo, nas questões éticas, considerando as perspetivas feministas e raciais (Jones et al., 2004; Taylor \& Trujillo, 2001). As perspetivas feministas têm em conta a marginalização das mulheres no mercado de trabalho; considerando o estabelecimento de relações de poder que desvalorizavam as mesmas. Por sua vez, as perspetivas raciais analisam o domínio de uma raça maioritária e a desvalorização e silêncio das minorias, no seio organizacional (Aschcraft \& Mumby, 2004; Miller, 2012; Schockley-Zalabak, 2012).

Importa referir os 7 aspetos fundamentais sobre os quais se baseia o paradigma dialógico:

A centralidade do discurso - a comunicação passa a ser uma forma de explicar as organizações e as suas atividades;

A fragmentação de identidades - o próprio ser humano tende a ser fragmentado, sendo que o discurso constitui-se como mais heterogéneo, podendo existir uma identidade fluída e conflitual;

A crítica da filosofia da presença - a linguagem torna-se essencial para a produção de objetos. Contudo, esta depende dos contextos social e histórico nos quais se encontram os indivíduos que concretizam a sua interpretação. Portanto, não tem de representar a realidade. Terá de considerar-se, simultaneamente, um entendimento das pessoas e da realidade;

Perda de fundações e das macro narrativas - contra as fundações das "verdades absolutas", como o Marxismo ou o funcionalismo;

A ligação entre o conhecimento e o poder - produção de conhecimento e verdade em sistemas de domínio. Foi o que criou o conceito de disciplina;

A hiperrealidade - as novas tecnologias e os fenómenos de mass media, levam a uma abertura relativamente a elementos externos;

A investigação como algo resistente e indeterminado - diferencia-se, bastante, dos discursos tradicionais, uma vez que tem em conta a área das ciências sociais, a produção de discórdia e de novas formas do conhecimento. Ou seja, tem em conta o papel que a investigação assume, em processos de mudança;

Abertura das ciências sociais modernas - defendendo-se que deverá ser alvo de mudança. 
Face aos aspetos acima mencionados consegue perceber-se que o paradigma dialógico considera os paradigmas positivista e interpretativo como simplistas, uma vez que estes não têm em conta as rápidas mudanças dos contextos e a multiplicidade de significados. Nesse sentido, os teóricos defensores do paradigma dialógico passam a colocar questões como: Como é que os múltiplos significados e as múltiplas interpretações podem influenciar múltiplos e diversos comportamentos? Face ao exposto, torna-se relevante mencionar que o método de análise do paradigma dialógico relaciona-se com aquilo que se designa por desconstrução. Ou seja, este paradigma baseiase em aspetos como: valores de partilha de poder, empowerment (i.e., empoderamento), interdependência, múltiplas interpretações de eventos diários (Schockley-Zalabak, 2012). Dentro dessa desconstrução, alguns autores defendem a existência de duas tendências. Por um lado, as meta-análises, os documentos organizacionais e a investigação organizacional. Por outro lado, os métodos associados à investigação empírica (Taylor \& Trujillo, 2001).

Conclui-se, então que, os aspetos acima frisados, que caraterizam o paradigma dialógico terão um impacto na qualidade da comunicação organizacional, e nas consequentes tomadas de decisão e direções das linhas de investigação.

\section{CONCLUSÃo}

Os diferentes paradigmas que estudam o conceito de comunicação organizacional têm vindo a surgir de forma fragmentada, uma vez que emergem da prática para a academia (Oliveira \& Ruão, 2013). Nesse sentido, perceber a evolução da comunicação organizacional enquanto disciplina torna-se fundamental para que os investigadores possam situar-se e escolher as suas próprias linhas de investigação. O presente trabalho permitiu-nos a identificação de quatro paradigmas principais, sendo estes: o paradigma positivista; o paradigma interpretativo; o paradigma crítico e o paradigma dialógico.

De forma genérica, tornou-se possível concluir que o paradigma positivista considera o estudo da comunicação organizacional e da sua relação com a eficácia organizacional (Mumby \& Stohl, 1996; Ruão, 1999; Spence \& Baker, 2007). Nesse sentido, este paradigma tem em conta modelos que considerem, essencialmente, aspetos relacionados com a formação de competências comunicativas, que possam contribuir para a eficiência das organizações (Red- 
ding \& Tompkins, 1988). Por sua vez, o paradigma interpretativo, considera que cada organização corresponde a uma realidade específica, desenvolvida através de conversas, histórias, ritos, rituais e outras atividades diárias (Deetz, 2001). Neste caso, a resolução de problemas será dada através da afirmação grupal e de uma aculturação social (Blair, Roberts \& McKecnie, 1985; Taylor \& Trujillo, 2001). O paradigma crítico encara as organizações como espaços de cariz político, sendo consideradas questões relacionadas com a hegemonia e com as assimetrias de poder. Este paradigma sugere, então, uma análise das dinâmicas de poder e das questões políticas, aquando do estudo da comunicação organizacional, considerando que estes exercícios de poder podem acarretar consequências negativas para as tomadas de decisão, gerando-se conflitos organizacionais (Alvesson \& Aschcraft, 2009, Ruão, 2008). Finalmente, o paradigma dialógico, ficou conhecido por rejeitar as noções de autoridade e poder, estabilidade de significados e conceitos de eficácia; representando uma alienação relativamente ao passado, um ceticismo acerca das estruturas de autoridade, ambiguidade de significados e cultura de massas (SchockleyZalabak, 2012).

A perspetiva histórica da comunicação organizacional, torna-se útil para a compreender como é que a mesma poderá constituir-se como um campo teórico autónomo, atualmente. Isto porque a investigação em comunicação organizacional não tem vindo a assumir uma identidade clara. O quadro atual reflete todo o percurso acima mencionado, encontrando-se marcado, essencialmente, pelo movimento interpretativo do século XX (Taylor \& Trujillo, 2001). Nesse sentido, sugere-se uma amálgama de paradigmas, adequandose, cada um deles, ao contexto organizacional específico que se pretende estudar. Conclui-se, então que, o futuro da comunicação organizacional, tal como o seu passado, terá em conta a implicação de vários métodos, teorias ecléticas e diferentes pressupostos que deverão ser considerados.

\section{REFERÊNCIAS}

Alvesson, M. \& Ashcraft, K. L. (2009). Critical methodology in management and organizational research. In D. A. Buchannan \& A. Bryman (Eds). The sage handbook of organizational research methods (pp. 61-77). Los Angeles, CA: Sage Publications. 
Ashcraft, K. L. \& Mumby, D. K. (2004). Reworking gender: a feminist communicology of organization. Thousand Oaks, CA: Sage Publications.

Blair, R.; Roberts, K. \& McKecnie, P. (1985). Vertical and network communications in organizations: the present and the future. In R. D. McPhee \& P. $\mathrm{K}$. Tompkins (eds). Organizational communication: traditional themes and new directions (pp. 55-77). U.S.A: Sage Publications.

Bouzon, A. (2013). Theoretical approaches in organizational and strategic communication: a review of the french academic literature. In G. Gonçalves, I Somerville \& A. Melo (eds). Organizational and strategic communication research: european perspetives (pp. 23-45). Covilhã: Livros Labcom.

Deetz, S. (2001). Conceptual foundations. In F. M. Jablin \& L. L. Putnam (Eds). The new handbook of organizational communication: advances in theory, research and methods (pp. 3-46). Thousand Oaks: Sage Publications.

Jones, E.; Watson, B.; Gardner, J. \& Gallois, C. (2004). Organizational communication: challenges for the new century. International Communication Association, 54: 722-750.

Krone, K. (2007). Trends in organizational communication research: sustaining the discipline, sustaining ourselves. Communication Studies, 56 (1): 95-105.

Miller, K. (2001). Quantitative research methods. In F. M. Jablin \& L. L. Putnam (Eds). The new handbook of organizational communication: advances in theory, research and methods (pp. 137-160). Thousand Oaks: Sage Publications.

Miller, K. (2012). Organizational communication: approaches and processes. Boston: Wadsworth, Cengage Learning.

Mumby, D. (2001). Power and politics. In F. M. Jablin \& L. L. Putnam (Eds). The new handbook of organizational communication: advances in theory, research and methods (pp. 585-623). Thousand Oaks: Sage Publications.

Mumby, D. K. \& Stohl, C. (1996). Disciplining organizational communication studies. Management Communication Quarterly, 10: 50-72. 
Oliveira, E. \& Ruão, T. (2013). Os quatro paradigmas da comunicação estratégica e o ensino em Portugal. Atas do VIII Congresso da SOPCOM. Lisboa: Escola Superior de Comunicação Social - Instituto Politécnico de Lisboa.

Putnam, L. \& Pacanowsky, M. (eds) (1983). Communication and organizations: an interpretative approach. Beverly Hills, CA: Sage Publications.

Redding, C. (1985). The emergence of organizational communication as a field of study. In R. D. McPhee \& P. K. Tompkins. Organizational communication: traditional themes and new directions (pp. 15-54). U.S.A: Sage Publications.

Redding, C. \& Tompkins, P. (1988). Organizational communication: past and presente tenses. In G. M. Goldhaber \& G. A. Barnett (eds). Handbook of organizational communication (pp. 5-33). Norwood: Ablex Publishing.

Ruão, T. (1999). A Comunicação organizacional e a gestão de recursos humanos: evolução e actualidade. Cadernos do Noroeste,12(1-2): 179-194.

Ruão, T. (2004). O estado da arte em comunicação organizacional. 19002000: um século de investigação. Atas do III Congresso da SOPCOM, VI LUSOCOM e II Ibérico. Covilhã: Universidade da Beira Interior.

Ruão, T. (2008). A Comunicação organizacional e os fenómenos de identidade: a aventura comunicativa da formação da Universidade do Minho, 1974-2006. Tese de Doutoramento, Universidade do Minho, Braga, Portugal.

Schockley-Zalabak (2012). Fundamentals of organizational communication: knowledge, sensitivity, skills, values ( $\left.8^{\circ} \mathrm{ed}\right)$. Colorado: Pearson Education.

Spence, P. R. \& Baker, C. R. (2007). State of the method: examination of levels of analysis, methodology, representation and setting in current organizational communication research. Journal of the Northwest Communication Association, 36: 111-124.

Taylor, B. \& Trujillo, N. (2001). Qualitative research methods. In F. M. Jablin \& L. L. Putnam (Eds). The new handbook of organizational communication: advances in theory, research and methods (pp. 161-196). Thousand Oaks: Sage Publications. 
Taylor, J. (1993). Rethinking the theory of organizational communication: how to read an organization. New Jersey: Ablex Publishing.

Wrench, J. S. (2013). Communicating within the modern workplace: challenges and prospects. In J. S. Wrench (Ed). Workplace communication for the 21st Century: tools and strategies that impact Bottom line. CA: Praeger.

Wrench, J. \& Punyanunt-Carter, N. (2012). An Introduction to organizational communication. U.S.A: Sage Publications.

Yanow, D. \& Ybema, S. (2009). Interpretivism in organizational research: on elephants and blind researchers. In. D. A. Buchannan \& A. Bryman (Eds). The sage handbook of organizational research methods (pp. 3960). Los Angeles, CA: Sage Publications. 